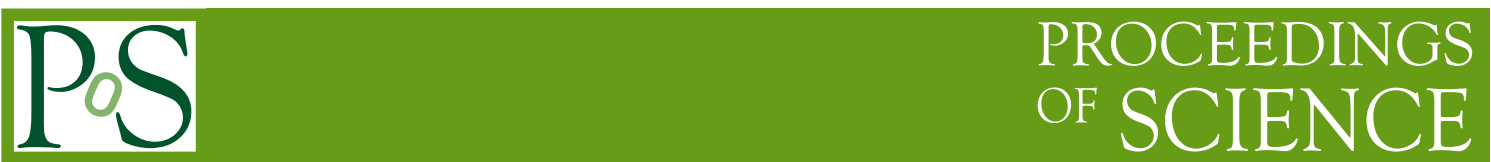

\title{
Electroweak precision tests in the LHC era and beyond
}

\author{
Ayres Freitas* \\ Pittsburgh Particle-physics Astro-physics \& Cosmology Center (PITT-PACC), Department of \\ Physics \& Astronomy, University of Pittsburgh, Pittsburgh, PA 15260, USA \\ E-mail: afreitas@pitt.edu
}

The current status of electroweak precision tests after the discovery of the Higgs boson is reviewed, both from a phenomenological and from a theoretical point of view. Predictions for all $Z$-pole quantities are now available at the complete fermionic two-loop order within the Standard Model. The calculation of these corrections is described based on the example of the total $Z$-boson width. Finally, an outlook on the experimental improvements and theoretical challenges for a future high-luminosity $e^{+} e^{-}$collider is given.

Loops and Legs in Quantum Field Theory - LL 2014,

27 April - 2 May 2014

Weimar, Germany

${ }^{*}$ Speaker. 


\section{Introduction}

With the recent discovery of the Higgs boson [1] and first measurements of its properties, the Standard Model (SM) has reached an unprecedented level of experimental confirmation. All free parameters of the SM have been now measured directly, and the agreement with indirect predictions from electroweak precision observables is highly non-trivial. A global electroweak SM fit including direct and indirect observables gives a high p-value of about 20\%, see e.g. Ref. [2]. For instance, the direct measurements for the top-quark mass and $W$-boson mass are $m_{\mathrm{t}}=173.24 \pm 0.81 \mathrm{GeV}$ [3] and $M_{\mathrm{W}}=80385 \pm 15 \mathrm{MeV}$ [4], respectively, while the fit to precision data yields $m_{\mathrm{t}}=177.0 \pm$ $2.1 \mathrm{GeV}$ and $M_{\mathrm{W}}=80358 \pm 7 \mathrm{MeV}$, see also Fig. 1 .

The electroweak precision fit uses inputs from a variety of sources, most notably the measurement of the muon decay constant (which leads to the strongest indirect constraint on $M_{\mathrm{W}}$ ) and properties of the Z-boson from measurements of $e^{+} e^{-}$collisions at $\sqrt{s} \approx M_{\mathrm{Z}}$ (which are very sensitive to $m_{\mathrm{t}}$ and the Higgs boson mass $M_{\mathrm{H}}$ ). These quantities have been measured with uncertainties of $\mathscr{O}(0.1 \%)$ or less. To match this precision on the theory side, one- and two-loop radiative corrections, as well as dominant higher-order contributions must be included.

Due to the efforts of several groups over many years, complete two-loop corrections are available for $M_{\mathrm{W}}$ [5] and the effective leptonic weak mixing angle $\sin ^{2} \theta_{\text {eff }}^{\ell}[6]$, which is extracted from the $Z$-pole left-right and forward-backward asymmetries. For the $Z$-boson width $\left(\Gamma_{\mathrm{Z}}\right)$ and branching ratios, and the hadronic pole cross-section $\sigma_{\text {had }}^{0} \equiv \sigma\left[e^{+} e^{-} \rightarrow Z \rightarrow\right.$ hadrons], the $\mathscr{O}\left(\alpha \alpha_{\mathrm{s}}\right)$ [7] and $\mathscr{O}\left(N_{f} \alpha^{2}\right)$ [8] corrections have been computed, where $N_{f}$ stands for "fermionic" contributions from diagrams with at least one closed fermion loops, which are expected to be dominant compared to the "bosonic" two-loop corrections. In addition, universal leading 3- and 4-loop corrections for large values of $m_{\mathrm{t}}$, of $\mathscr{O}\left(\alpha_{\mathrm{t}} \alpha_{\mathrm{s}}^{2}\right), \mathscr{O}\left(\alpha_{\mathrm{t}}^{2} \alpha_{\mathrm{s}}\right), \mathscr{O}\left(\alpha_{\mathrm{t}}^{3}\right)$ and $\mathscr{O}\left(\alpha_{\mathrm{t}} \alpha_{\mathrm{s}}^{3}\right)$, are known for all aforementioned observables [9]. Here the abbreviation $\alpha_{\mathrm{t}}=\alpha m_{\mathrm{t}}^{2}$ has been used. As evident from Tab. 1, the theory errors from missing higher-order corrections are safely below current experimental uncertainties.

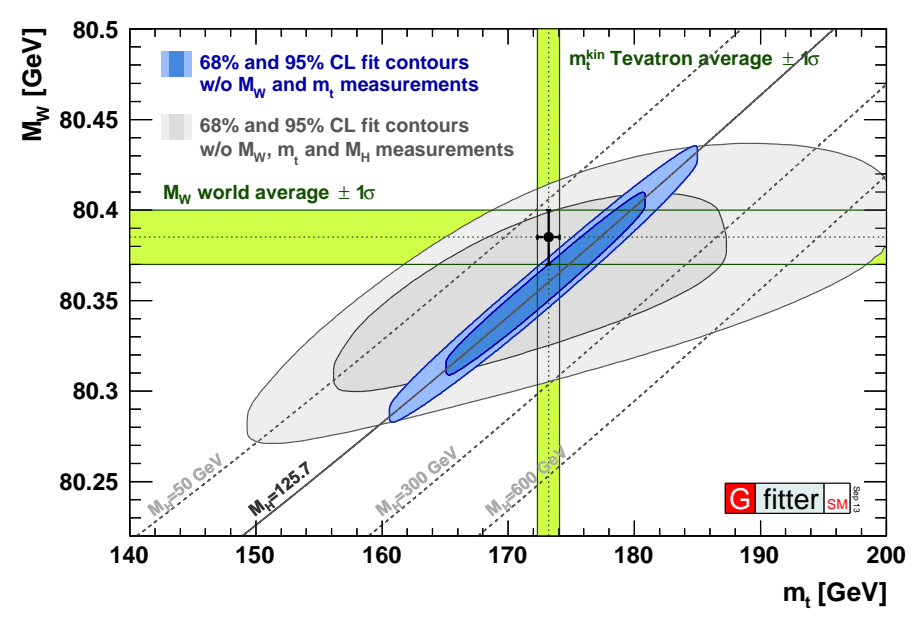

Figure 1: Comparison of direct determinations (green) of $m_{\mathrm{t}}$ and $M_{\mathrm{W}}$ with the indirect determination from electroweak precision data before (gray) and after (blue) the Higgs discovery. From Ref. [2]. 


\begin{tabular}{lccccc}
\hline & $M_{\mathrm{W}}$ & $\Gamma_{\mathrm{Z}}$ & $\sigma_{\text {had }}^{0}$ & $R_{\mathrm{b}}$ & $\sin ^{2} \theta_{\text {eff }}^{\ell}$ \\
\hline Exp. error & $15 \mathrm{MeV}$ & $2.3 \mathrm{MeV}$ & $37 \mathrm{pb}$ & $6.6 \times 10^{-4}$ & $1.6 \times 10^{-4}$ \\
Theory error & $4 \mathrm{MeV}$ & $0.5 \mathrm{MeV}$ & $6 \mathrm{pb}$ & $1.5 \times 10^{-4}$ & $0.5 \times 10^{-4}$ \\
\hline
\end{tabular}

Table 1: Current experimental errors and theory uncertainties for the SM prediction of some of the most important electroweak precision observables. Here $R_{b} \equiv \Gamma[Z \rightarrow b \bar{b}] / \Gamma[Z \rightarrow$ hadrons $]$.

\section{Z-boson width at two loops}

As a concrete example for the electroweak two-loop corrections to electroweak precision observables, this section will discuss the calculation of the $\mathscr{O}\left(N_{f} \alpha^{2}\right)$ contribution to the (partial) $Z$-boson width(s). The total $Z$-width is defined through the imaginary part of the complex pole of the $Z$-boson propagator,

$$
s_{0}=M_{\mathrm{Z}}^{2}-i M_{\mathrm{Z}} \Gamma_{\mathrm{Z}} .
$$

This definition leads to a Breit-Wigner function with constant width near the Z-pole, $\sigma \propto$ $\left|s-s_{0}\right|^{-2}=\left[\left(s-M_{\mathrm{Z}}^{2}\right)^{2}+M_{\mathrm{Z}}^{2} \Gamma_{\mathrm{Z}}^{2}\right]^{-1}$. Note that this differs from the Breit-Wigner function with a running width used in the experimental analyses, so that one has to include a finite shift when relating $M_{\mathrm{Z}}$ and $\Gamma_{\mathrm{Z}}$ to the reported measured values:

$$
M_{\mathrm{Z}}=M_{\mathrm{Z}}^{\mathrm{exp}}-34.1 \mathrm{MeV}, \quad \Gamma_{\mathrm{Z}}=\Gamma_{\mathrm{Z}}^{\mathrm{exp}}-0.9 \mathrm{MeV} .
$$

Expanding (2.1) up to next-to-next-to-leading order (NNLO) and using the power counting $\Gamma_{\mathrm{Z}} \sim$ $\mathscr{O}(\alpha) M_{\mathrm{Z}}$, the result for $\Gamma_{\mathrm{Z}}$ can be written as $[8]^{1}$

$$
\Gamma_{\mathrm{Z}}=\frac{1}{M_{\mathrm{Z}}} \operatorname{Im} \Sigma_{\mathrm{Z}}\left(s_{0}\right)=\frac{1}{M_{\mathrm{Z}}}\left[\frac{\operatorname{Im} \Sigma_{\mathrm{Z}}}{1+\operatorname{Re} \Sigma_{\mathrm{Z}}^{\prime}}\right]_{s=M_{\mathrm{Z}}^{2}}+\mathscr{O}\left(\Gamma_{\mathrm{Z}}^{3}\right),
$$

where $\Sigma_{Z}$ is the $Z$ self-energy. Using the optical theorem, the imaginary part of the self-energy can be related to the decay process $Z \rightarrow f \bar{f}$, resulting in

$$
\Gamma_{\mathrm{Z}}=\sum_{f} \Gamma_{f}, \quad \Gamma_{f}=\frac{N_{c}^{f} M_{\mathrm{Z}}}{12 \pi}\left[\mathscr{R}_{V}^{f} F_{V}^{f}+\mathscr{R}_{A}^{f} F_{A}^{f}\right]_{s=M_{\mathrm{Z}}^{2}}, \quad F_{V}^{f} \approx \frac{\left|v_{f}\right|^{2}}{1+\operatorname{Re} \Sigma_{\mathrm{Z}}^{\prime}}, \quad F_{A}^{f} \approx \frac{\left|a_{f}\right|^{2}}{1+\operatorname{Re} \Sigma_{\mathrm{Z}}^{\prime}},
$$

where $N_{c}^{f}=3(1)$ for quarks (leptons). Here the functions $\mathscr{R}_{V, A}^{f}$ have been introduced, which capture effects from final-state QED and QCD corrections. They are known up to $\mathscr{O}\left(\alpha_{\mathrm{s}}^{4}\right), \mathscr{O}\left(\alpha \alpha_{\mathrm{s}}\right)$ and $\mathscr{O}\left(\alpha^{2}\right)$ in the limit of massless fermions, while mass corrections are known up to three-loop order [10]. The electroweak corrections are contained in $\Sigma_{Z}^{\prime}$ and the effective $Z f \bar{f}$ vector and axial-vector couplings $v_{f}$ and $a_{f}$. Note that $v_{f}$ and $a_{f}$ include contributions from photon- $Z$ mixing. Eq. (2.4) is accurate up to NNLO.

For the calculation of the fermionic electroweak $\mathscr{O}\left(\alpha^{2}\right)$ corrections, Feynman diagrams have been generated with FeynArts 3.3 [11]. In addition to the diagrams for the $Z \rightarrow f \bar{f}$ vertex corrections, one also needs two-loop self-energy diagrams for the on-shell renormalization [12]. In the on-shell renormalization scheme used here, particle masses are defined through the (complex)

\footnotetext{
${ }^{1}$ Here a term $\propto \operatorname{Im} \Sigma_{Z}^{\prime \prime}$ has been omitted, since $\operatorname{Im} \Sigma_{Z}^{\prime \prime}=0$ at leading order for massless final-state fermions.
} 
pole of the propagators. For the electroweak NNLO contributions, only $M_{\mathrm{Z}}, M_{\mathrm{W}}, M_{\mathrm{H}}$ and $m_{\mathrm{t}}$ are taken non-zero, while all other fermion masses have been neglected (except for the final-state QED and QCD corrections, where also non-vanishing bottom, charm and tau masses have been taken into account). The electromagnetic coupling is renormalized in the Thomson limit, i.e. for zero momentum exchange, while $\alpha_{\mathrm{s}}$ is defined in the $\overline{\mathrm{MS}}$ scheme.

Two-loop integrals with a sub-loop self-energy bubble can be easily reduced to a small set of master integrals using a generalization of the Passarino-Veltman method [13] and integrationby-parts identities [14]. These master integrals can be evaluated numerically in terms of simple one-dimensional integrations [15]

$$
\overbrace{m_{1}}^{m_{N-1}}=-2 \int_{\left(m_{N}+m_{N+1}\right)^{2}}^{\infty} d s \operatorname{Im}\left[B_{0}\left(s, m_{N}^{2}, m_{N+1}^{2}\right)\right] I^{(1)}\left(p_{1}, \ldots, p_{N} ; s, m_{1}^{2}, \ldots, m_{N-1}^{2}\right),
$$

where $B_{0}$ is the usual one-loop two-point integral, while $I^{(1)}\left(\left\{p_{i}\right\} ;\left\{m_{j}\right\}\right)$ is a general scalar oneloop integral with momenta $\left\{p_{i}\right\}$ and masses $\left\{m_{j}\right\}$.

For two-loop vertex integrals with a triangle sub-loop, a technique based on numerical integration over Feynman parameters has been used [16]. Before the numerical integral can be carried out, potential divergences must be subtracted. The global ultraviolet (UV) divergence of an integral $I^{(2)}=\int d^{d} k_{1} d^{d} k_{2} G\left(k_{1}, k_{2},\left\{p_{i}\right\}\right)$ is extracted by the operation

$$
I^{(2)}=\int d^{d} k_{1} d^{d} k_{2}\left[G\left(k_{1}, k_{2},\left\{p_{i}\right\}\right)-G\left(k_{1}, k_{2},\{0\}\right)\right]+\int d^{d} k_{1} d^{d} k_{2} G\left(k_{1}, k_{2},\{0\}\right) \equiv I_{\mathrm{gs}}^{(2)}+I_{\mathrm{glob}}^{(2)}
$$

Then $I_{\mathrm{gs}}^{(2)}$ is free of global UV singularities, while $I_{\text {glob }}^{(2)}$ is a two-loop vacuum integral, which can be solved analytically [17]. $I_{\mathrm{gs}}^{(2)}$ may still contain sub-loop UV singularities. After introducing Feynman parameters for the divergent sub-loop (assumed to be the $k_{1}$ loop), shifting the loop momentum, and appropriately canceling $k_{1}$ between the numerators and denominator, the integral has the form

$$
I_{\mathrm{gs}}^{(2)}=\int_{0}^{1} d x_{1} \ldots d x_{m-1} \int d^{d} k_{1} d^{d} k_{2}\left[\frac{C_{1}}{\left[k_{1}^{2}-A\right]^{m}}+\frac{C_{2}}{\left[k_{1}^{2}-A\right]^{m-1}}+\cdots+\frac{C_{m-1}}{\left[k_{1}^{2}-A\right]^{2}}\right],
$$

where $A$ and the $C_{i}$ can depend on $k_{2}$ but not $k_{1}$. The sub-loop UV divergence is contained in the last term $\propto C_{m-1}$ and can be removed with the subtraction term $I_{\text {sub }}^{(2)}=\int_{0}^{1} d x_{1} \ldots d x_{m-1} \int d^{d} k_{1} d^{d} k_{2} \frac{C_{m-1}}{\left[k_{1}^{2}-\mu^{2}\right]^{2}}$, where $\mu^{2}$ is a suitably chosen constant parameter. Since $\mu^{2}$ is constant, the $k_{1}$ and Feynman parameter integration of $I_{\text {sub }}^{(2)}$ can be trivially performed analytically, whereas $I_{\mathrm{gs}}^{(2)}-I_{\text {sub }}^{(2)}$ is now UV-finite.

Infrared divergences can also be handled with suitable subtraction terms [16], but for the present calculation a photon mass has been used instead, see Refs. [8, 18] for details.

After also introducing Feynman parameters for the $k_{2}$ loop, the Feynman parameter integration of the subtracted integral is performed numerically. In the presence of physical thresholds, the integrand will in general contain additional singularities where the denominator term $A$ vanishes. These internal singularities are formally integrable but lead to difficulties for standard integration 
algorithms. However, they can be avoided with a complex variable transformation [19]

$$
x_{i}=z_{i}-\left.i \lambda z_{i}\left(1-z_{i}\right) \frac{\partial A}{\partial x_{i}}\right|_{\vec{x}=\vec{z}}, \quad 0 \leq z_{i} \leq 1,
$$

so that

$$
A(\vec{x})=A(\vec{z})-i \lambda \sum_{i} z_{i}\left(1-z_{i}\right)\left(\frac{\partial A}{\partial x_{i}}\right)_{\vec{x}=\vec{z}}^{2}+\mathscr{O}\left(\lambda^{2}\right) .
$$

For sufficiently small $\lambda$, one can see that $A(\vec{x})$ will never vanish as long as there is no point where $A(\vec{z})=\nabla A(\vec{z})=0$ at the same time. More details of the calculation can be found in Ref. [8].

\section{Z-boson width: Results}

In the following, numerical results for the form factors $F_{V, A}^{f}$ from (2.4) will be presented. For this purpose, the vector and axial-vector components of the electroweak one-loop and fermionic two-loop corrections were combined with the $\mathscr{O}\left(\alpha \alpha_{\mathrm{s}}\right)$ contributions from Ref. [7], which also had to be split into vector and axial-vector parts. Furthermore, the universal leading higher-order corrections of $\mathscr{O}\left(\alpha_{\mathrm{t}} \alpha_{\mathrm{s}}^{2}\right), \mathscr{O}\left(\alpha_{\mathrm{t}}^{2} \alpha_{\mathrm{s}}\right), \mathscr{O}\left(\alpha_{\mathrm{t}}^{3}\right)$ and $\mathscr{O}\left(\alpha_{\mathrm{t}} \alpha_{\mathrm{s}}^{3}\right)$ [9] have been included. The results are expressed in terms of the on-shell masses $M_{\mathrm{Z}}, M_{\mathrm{H}}$ and $m_{\mathrm{t}}$, the $\overline{\mathrm{MS}}$ strong coupling constant $\alpha_{\mathrm{s}}\left(M_{\mathrm{Z}}\right)$, and the shift $\Delta \alpha$ of the electromagnetic coupling between the scales $q^{2}=0$ and $M_{Z}^{2}, \Delta \alpha=1-$ $\alpha(0) / \alpha\left(M_{\mathrm{Z}}^{2}\right)$. Note that the $W$-mass is computed using the SM prediction for $M_{\mathrm{W}}$ [5].

The results can be conveniently expressed in terms of a simple parametrization formula

$$
\begin{aligned}
F_{X}^{f}= & F_{0}+a_{1} L_{\mathrm{H}}+a_{2} L_{\mathrm{H}}^{2}+a_{3} \Delta_{\mathrm{H}}+a_{4} \Delta_{\mathrm{H}}^{2}+a_{5} \Delta_{\mathrm{t}}+a_{6} \Delta_{\mathrm{t}}^{2}+a_{7} \Delta_{\mathrm{t}} L_{\mathrm{H}} \\
& +a_{8} \Delta_{\alpha_{\mathrm{s}}}+a_{9} \Delta_{\alpha_{\mathrm{s}}} L_{\mathrm{H}}+a_{10} \Delta_{\alpha_{\mathrm{s}}} \Delta_{\mathrm{t}}+a_{11} \Delta_{\alpha}+a_{12} \Delta_{\mathrm{Z}}, \\
L_{\mathrm{H}}= & \log \frac{M_{\mathrm{H}}}{125.7 \mathrm{GeV}}, \quad \Delta_{\mathrm{H}}=\frac{M_{\mathrm{H}}}{125.7 \mathrm{GeV}}-1, \quad \Delta_{\mathrm{t}}=\left(\frac{m_{\mathrm{t}}}{173.2 \mathrm{GeV}}\right)^{2}-1, \\
\Delta_{\alpha_{\mathrm{s}}}= & \frac{\alpha_{\mathrm{s}}\left(M_{\mathrm{Z}}\right)}{0.1184}-1, \quad \Delta_{\alpha}=\frac{\Delta \alpha}{0.059}-1, \quad \Delta_{\mathrm{Z}}=\frac{M_{\mathrm{Z}}}{91.1876 \mathrm{GeV}}-1 .
\end{aligned}
$$

Table 2 shows the values of the coefficients obtained from a fit of (3.1) to the full result.

Results for the complete observables, i.e. the total and partial widths including the final state radiation functions $\mathscr{R}_{V, A}^{f}$, have been presented elsewhere [8].

\section{Outlook}

Electroweak precision observables are a very useful tool for accurate indirect tests of the SM and setting stringent constraints on new physics. At the current level of experimental precision, they are sensitive to one- and two-loop and even leading higher-order effects. Nevertheless, due to tremendous efforts by many groups, the error of the theoretical predictions within the SM has been reduced comfortably below the experimental uncertainty for the most relevant quantities, see Tab. 1. The LHC experiments are expected to provide interesting independent determinations of $M_{\mathrm{W}}$ and $\sin ^{2} \theta_{\text {eff }}^{\ell}$ [20], but the overall experimental precision for these quantities will not improve markedly. 


\begin{tabular}{lrrrcrrr}
\hline Form factor & \multicolumn{1}{c}{$F_{0}$} & \multicolumn{1}{c}{$a_{1}$} & \multicolumn{1}{c}{$a_{2}$} & \multicolumn{1}{c}{$a_{3}$} & \multicolumn{1}{c}{$a_{4}$} & \multicolumn{1}{c}{$a_{5}$} & \multicolumn{1}{c}{$a_{6}$} \\
\hline$F_{V}^{\ell}\left[10^{-5}\right]$ & 19.84 & -1.012 & -0.1654 & 0.1467 & -0.00440 & 7.449 & 1.47 \\
$F_{A}^{\ell}\left[10^{-5}\right]$ & 3446.44 & -3.2302 & -2.4615 & 2.1482 & -0.05666 & 25.856 & -2.59 \\
$F_{V, A}^{v}\left[10^{-5}\right]$ & 3456.63 & -3.2844 & -2.4733 & 2.1561 & -0.05674 & 26.075 & -2.69 \\
$F_{V}^{u, c}\left[10^{-5}\right]$ & 505.88 & -4.3145 & -1.013 & 0.9632 & -0.02295 & 23.31 & -1.79 \\
$F_{A}^{u, c}\left[10^{-5}\right]$ & 3448.32 & -3.2438 & -2.4645 & 2.1502 & -0.05668 & 25.982 & -2.66 \\
$F_{V}^{d, s}\left[10^{-5}\right]$ & 1650.61 & -5.0642 & -1.7806 & 1.6318 & -0.04038 & 29.384 & -3.15 \\
$F_{A}^{d, s}\left[10^{-5}\right]$ & 3450.99 & -3.2528 & -2.4664 & 2.1514 & -0.05669 & 25.824 & -2.63 \\
$F_{V}^{b}\left[10^{-5}\right]$ & 1620.96 & -4.7874 & -1.7196 & 1.5915 & -0.04005 & -3.582 & -1.97 \\
$F_{A}^{b}\left[10^{-5}\right]$ & 3408.18 & -2.9546 & -2.4005 & 2.1073 & -0.05631 & -21.79 & -1.02 \\
\hline
\end{tabular}

\begin{tabular}{lrrrrrrc}
\hline Form factor & \multicolumn{1}{c}{$a_{7}$} & \multicolumn{1}{c}{$a_{8}$} & \multicolumn{1}{c}{$a_{9}$} & \multicolumn{1}{c}{$a_{10}$} & \multicolumn{1}{c}{$a_{11}$} & $a_{12}$ & max. dev. \\
\hline$F_{V}^{\ell}\left[10^{-5}\right]$ & -0.278 & -0.13 & -0.17 & 2.6 & -42.7 & 1373 & 0.05 \\
$F_{A}^{\ell}\left[10^{-5}\right]$ & 0.0010 & -3.80 & -0.44 & 7.06 & -1.4 & 6915 & 0.07 \\
$F_{V, A}^{v}\left[10^{-5}\right]$ & -0.0005 & -3.86 & -0.44 & 7.06 & -1.2 & 6943 & 0.07 \\
$F_{V}^{u, c}\left[10^{-5}\right]$ & -0.067 & -3.88 & -0.37 & 6.07 & -145.2 & 5639 & 0.05 \\
$F_{A}^{u, c}\left[10^{-5}\right]$ & 0.0004 & -4.79 & -0.43 & 6.97 & -1.3 & 6922 & 0.07 \\
$F_{V}^{d, s}\left[10^{-5}\right]$ & 0.028 & -5.52 & -0.45 & 7.55 & -131.2 & 7491 & 0.07 \\
$F_{A}^{d, s}\left[10^{-5}\right]$ & 0.0036 & -4.96 & -0.43 & 6.97 & -1.1 & 6927 & 0.07 \\
$F_{V}^{b}\left[10^{-5}\right]$ & 0.334 & -1.28 & -0.50 & 11.9 & -130.6 & 7446 & 0.07 \\
$F_{A}^{b}\left[10^{-5}\right]$ & 0.340 & 0.96 & -0.49 & 13.0 & -1.7 & 6917 & 0.07 \\
\hline
\end{tabular}

Table 2: Coefficients for the parametrization formula (3.1) for the $Z f \bar{f}$ form factors. Within the ranges $70 \mathrm{GeV}<M_{\mathrm{H}}<1000 \mathrm{GeV}, 165 \mathrm{GeV}<m_{\mathrm{t}}<190 \mathrm{GeV}, \alpha_{\mathrm{s}}=0.1184 \pm 0.0050, \Delta \alpha=0.0590 \pm 0.0005$ and $M_{\mathrm{Z}}=91.1876 \pm 0.0084 \mathrm{GeV}$, the formula approximates the full result with maximal deviations given in the last column. When restricting oneself to the range $M_{\mathrm{H}}=125.7 \pm 2.5 \mathrm{GeV}$, the maximum deviation is reduced by a factor of more than 5 .

However, a future high-luminosity $e^{+} e^{-}$machine like ILC will substantially increase the experimental precision, thus posing a challenge for theorists to match this precision. In Table 3, the expected ILC precision is compared with the current and projected theory uncertainty for several observables. For the projection, it is assumed that the leading fermionic three-loop corrections of order $\mathscr{O}\left(N_{f}^{2} \alpha \alpha_{\mathrm{s}}\right), \mathscr{O}\left(N_{f} \alpha \alpha_{\mathrm{s}}\right), \mathscr{O}\left(N_{f}^{3} \alpha^{3}\right)$ and $\mathscr{O}\left(N_{f}^{2} \alpha^{3}\right)$ will be computed, where $N_{f}$ indicates the number of closed fermion loops. These contributions imply three-loop self-energies and threeloop vertices with sub-loop bubbles. The remaining theory error is estimated by approximating the perturbation series with a geometric series.

As evident from the table, it seems not preposterous to believe that the theory calculations can achieve a level of precision comparable or better than the expected ILC precision. In fact, for most quantities, the parametric error due to the uncertainty of input parameters will dominate over the perturbative theory error.

Acknowledgements: This work has been supported in part by the National Science Foundation under grant no. PHY-1212635. 


\begin{tabular}{lcccc}
\hline & $M_{\mathrm{W}}$ & $\Gamma_{\mathrm{Z}}$ & $R_{\mathrm{b}}$ & $\sin ^{2} \theta_{\text {eff }}^{\ell}$ \\
\hline ILC exp. error & $3 \ldots 5 \mathrm{MeV}$ & $\sim 1 \mathrm{MeV}$ & $1.5 \times 10^{-4}$ & $1.3 \times 10^{-5}$ \\
Current theory error & $4 \mathrm{MeV}$ & $0.5 \mathrm{MeV}$ & $1.5 \times 10^{-4}$ & $4.5 \times 10^{-5}$ \\
Projected theory error & $1 \mathrm{MeV}$ & $0.2 \mathrm{MeV}$ & $0.5 \ldots 1 \times 10^{-4}$ & $1.5 \times 10^{-5}$ \\
Parametric error for ILC & $2.6 \mathrm{MeV}$ & $0.5 \mathrm{MeV}$ & $<10^{-5}$ & $2 \times 10^{-5}$ \\
\hline
\end{tabular}

Table 3: Projected experimental errors of ILC running at $\sqrt{s} \approx M_{\mathrm{Z}}$ and $\sqrt{s} \approx 2 M_{\mathrm{W}}$ [21] and current and expected future theory uncertainties for the SM prediction for several important electroweak precision observables. The future theory errors are estimated under the assumption that $\mathscr{O}\left(N_{f}^{2} \alpha \alpha_{\mathrm{s}}\right), \mathscr{O}\left(N_{f} \alpha \alpha_{\mathrm{s}}\right), \mathscr{O}\left(N_{f}^{3} \alpha^{3}\right)$ and $\mathscr{O}\left(N_{f}^{2} \alpha^{3}\right)$ corrections will become available. The parametric error describes the uncertainty of the $\mathrm{SM}$ prediction due to uncertainties of input parameters: $\delta m_{\mathrm{t}}=100 \mathrm{MeV}, \delta \alpha_{\mathrm{s}}=0.001$ (from ILC), and $\delta M_{\mathrm{Z}}=2.1 \mathrm{MeV}$ (from LEP).

\section{References}

[1] G. Aad et al. [ATLAS Collaboration], Phys. Lett. B 716 (2012) 1 [arXiv:1207.7214 [hep-ex]]; S. Chatrchyan et al. [CMS Collaboration], Phys. Lett. B 716 (2012) 30 [arXiv:1207.7235 [hep-ex]].

[2] M. Baak et al., Eur. Phys. J. C 72 (2012) 2205 [arXiv:1209.2716 [hep-ph]], and updates at gfitter.desy.de.

[3] M. Muether et al. [Tevatron Electroweak Working Group and CDF and D $\varnothing$ Collaborations], arXiv:1305.3929 [hep-ex]; The ATLAS and CMS Collaborations, ATLAS-CONF-2013-102.

[4] S. Schael et al. [ALEPH and DELPHI and L3 and OPAL and LEP Electroweak Collaborations], Phys. Rept. 532 (2013) 119 [arXiv:1302.3415 [hep-ex]]; T. A. Aaltonen et al. [CDF and DØ Collaborations], Phys. Rev. D 88 (2013) 5, 052018 [arXiv:1307.7627 [hep-ex]].

[5] A. Freitas, W. Hollik, W. Walter and G. Weiglein, Phys. Lett. B 495 (2000) 338 [Erratum-ibid. B 570 (2003) 260] [hep-ph/0007091]; M. Awramik and M. Czakon, Phys. Rev. Lett. 89 (2002) 241801 [hep-ph/0208113], Phys. Lett. B 568 (2003) 48 [hep-ph/0305248]; A. Onishchenko and O. Veretin, Phys. Lett. B 551 (2003) 111 [hep-ph/0209010]; M. Awramik, M. Czakon, A. Freitas and G. Weiglein, Phys. Rev. D 69 (2004) 053006 [hep-ph/0311148].

[6] M. Awramik, M. Czakon, A. Freitas, G. Weiglein, Phys. Rev. Lett. 93 (2004) 201805 [hep-ph/0407317]; M. Awramik, M. Czakon and A. Freitas, Phys. Lett. B 642 (2006) 563 [hep-ph/0605339]; W. Hollik, U. Meier and S. Uccirati, Nucl. Phys. B 731 (2005) 213 [hep-ph/0507158], Nucl. Phys. B 765 (2007) 154 [hep-ph/0610312].

[7] A. Czarnecki and J. H. Kühn, Phys. Rev. Lett. 77 (1996) 3955 [hep-ph/9608366]; R. Harlander, T. Seidensticker and M. Steinhauser, Phys. Lett. B 426 (1998) 125 [hep-ph/9712228].

[8] A. Freitas, Phys. Lett. B 730 (2014) 50 [arXiv:1310.2256 [hep-ph]], JHEP 1404 (2014) 070 [arXiv:1401.2447 [hep-ph]].

[9] L. Avdeev, J. Fleischer, S. Mikhailov and O. Tarasov, Phys. Lett. B 336 (1994) 560 [Erratum-ibid. B 349 (1994) 597] [hep-ph/9406363]; K. G. Chetyrkin, J. H. Kühn and M. Steinhauser, Phys. Lett. B 351 (1995) 331 [hep-ph/9502291]; J. J. van der Bij, K. G. Chetyrkin, M. Faisst, G. Jikia and T. Seidensticker, Phys. Lett. B 498 (2001) 156 [hep-ph/0011373]; M. Faisst, J. H. Kühn, T. Seidensticker and O. Veretin, Nucl. Phys. B 665 (2003) 649 [hep-ph/0302275]; Y. Schröder and M. Steinhauser, Phys. Lett. B 622 (2005) 124 [hep-ph/0504055]; K. G. Chetyrkin, M. Faisst, J. H. Kühn, P. Maierhoefer and C. Sturm, Phys. Rev. Lett. 97 (2006) 102003 [hep-ph/0605201]; R. Boughezal and M. Czakon, Nucl. Phys. B 755 (2006) 221 [hep-ph/0606232]. 
[10] K. G. Chetyrkin, J. H. Kühn and A. Kwiatkowski, Phys. Rept. 277, 189 (1996); P. A. Baikov, K. G. Chetyrkin and J. H. Kühn, Phys. Rev. Lett. 101, 012002 (2008) [arXiv:0801.1821 [hep-ph]]; P. A. Baikov, K. G. Chetyrkin, J. H. Kühn and J. Rittinger, Phys. Rev. Lett. 108, 222003 (2012) [arXiv:1201.5804 [hep-ph]]; A. L. Kataev, Phys. Lett. B 287, 209 (1992).

[11] T. Hahn, Comput. Phys. Commun. 140, 418 (2001) [hep-ph/0012260].

[12] A. Freitas, W. Hollik, W. Walter and G. Weiglein, Nucl. Phys. B 632 (2002) 189 [Erratum-ibid. B 666 (2003) 305] [hep-ph/0202131].

[13] G. Weiglein, R. Scharf and M. Böhm, Nucl. Phys. B 416, 606 (1994) [hep-ph/9310358].

[14] K. G. Chetyrkin and F. V. Tkachov, Nucl. Phys. B 192, 159 (1981);

[15] S. Bauberger, F. A. Berends, M. Böhm and M. Buza, Nucl. Phys. B 434, 383 (1995); [hep-ph/9409388]; S. Bauberger and M. Böhm, Nucl. Phys. B 445, 25 (1995) [hep-ph/9501201].

[16] A. Freitas, JHEP 1207, 132 (2012) [Erratum-ibid. 1209, 129 (2012)] [arXiv:1205.3515 [hep-ph]].

[17] A. I. Davydychev and J. B. Tausk, Nucl. Phys. B 397 (1993) 123.

[18] M. Awramik, M. Czakon and A. Freitas, JHEP 0611, 048 (2006) [arXiv:hep-ph/0608099].

[19] Z. Nagy and D. E. Soper, Phys. Rev. D 74, 093006 (2006) [hep-ph/0610028].

[20] G. L. Bayatian et al. [CMS Collaboration], J. Phys. G 34 (2007) 995; G. Aad et al. [ATLAS Collaboration], arXiv:0901.0512 [hep-ex].

[21] R. Hawkings and K. Mönig, Eur. Phys. J. direct C 1 (1999) 8 [hep-ex/9910022]; M. Baak et al., arXiv:1310.6708 [hep-ph]. 